\section{DRL performance in hamsters with septal lesions}

\section{STEPHEN H. HOBBS and BRADFORD N. BUNNELL University of Georgia, Athens, Ga. 30601}

Bilateral septal lesions in male golden hamsters resulted in an initial postoperative response reduction on a DRL 20 -sec schedule of barpressing for saccharin solution reinforcement. The animals were not water deprived. When water deprivation was instituted, the lesioned animals showed significantly higher rates of responding than the controls. Fluid consumption tests indicated that the lesion effects were not due to changes in fluid intake or saccharin preference.
Septal lesions in rats usually produce deficits in the acquisition (Ellen, Wilson, \& Powell, 1964) and retention (Burkett \& Bunnell, 1966) of a differential reinforcement of low rate (DRL) schedule of barpressing. Under the DRL schedule, the $S$ is reinforced only for interresponse times (IRTs) which exceed some specified value. Harvey \& Hunt (1965) indicated that increased barpressing by rats with septal lesions might be due to increased motivation for water. Carey $(1967 \mathrm{a}, \mathrm{b})$ has presented data showing that the increased water consumption observed following septal lesions in rats is independent of the increased responding.

As an additional step in separating lesion-produced motivation and performance changes, the present study investigated the performance of hamsters with septal lesions on the DRL schedule. Matalka (1967) has shown that hamsters given bilateral septal lesions do not increase water consumption postoperatively as do rats.

\section{SUBJECTS}

Twelve adult male golden hamsters (Mesocricetus auratus) from Manor Farms (Manor Golden Fawn Strain) were housed in individual cages with food, water, and nesting materials available at all times, except as indicated below. All behavioral testing took place during light-on hours.

\section{APPARATUS}

Testing was conducted in two Lehigh Valley Electronics operant boxes (Model 1417), which were modified slightly to accommodate the $1 / 4$-in. hardware cloth, and sheet metal was inserted as a rear wall to reduce the inside floor dimensions. A mouse lever was inserted in each box to the left of the dipper. Electromechanical progr imming and recording equipment was located in an adjoining room.

\section{PROCEDURE}

All preoperative training was conducted with no water deprivation and with $.01 \mathrm{cc}$ of a $.1 \%$ solution of hamster. The floor was covered with sodium saccharin servirg as reinforcement. After the barpressing response had been acquired, the $\mathrm{Ss}$ were put on a DRL 20-sec schedule of reinforcement. Each daily DRL session lasted $30 \mathrm{~min}$. Preoperative DRL sessions continued until an animal reached a response/reinforcement (efficiency) ratio of 3.00 or less on 2 consecutive days or until the animal completed 35 sessions.

The Ss were matched with respect to their efficiency ratios and divided into two groups. Six Ss were given bilateral septal lesions. The control group consisted of two normals and four surgical controls (two with bilateral ablations of lateral cortex, one with bilateral ablation of medial cortex, and one in which the electrode was lowered to the level of the corpus callosum with no current being passed). DRL sessions were resumed for 20 days under conditions identical to those given preoperatively. Subsequently, after 3 days of exposure to a 22.5-h water-deprivation schedule, the Ss were run for five sessions under deprived conditions. Saccharin remained the reinforcement. Five additional nondeprived sessions were then given, beginning on the day following the last deprivation session.

Two-bottle saccharin preference tests were given on the 5 days prior to surgery and the last 5 days of the surgical recovery period. Positions of the bottles on the home cages were randomized.

\section{SURGERY AND HISTOLOGY}

Surgery was performed under sodium pentobarbital anesthesia on the fifth day following the last DRL session. Lesions of the septal area were made with a 30-ga stainless steel electrode, insulated except for $.75 \mathrm{~mm}$ at the tip, and a Grass RF lesion generator (Model LM3). A rectal electrode completed the circuit. With the head leveled according to the procedure of Smith \& Bodemer (1963), the coordinates for the septal lesions were: $6.00 \mathrm{~mm}$ anterior to bregma, $.50 \mathrm{~mm}$ lateral to both sides of the midline, and $4.20 \mathrm{~mm}$ below the surface of the cortex. The
Following a 14-day recovery period,

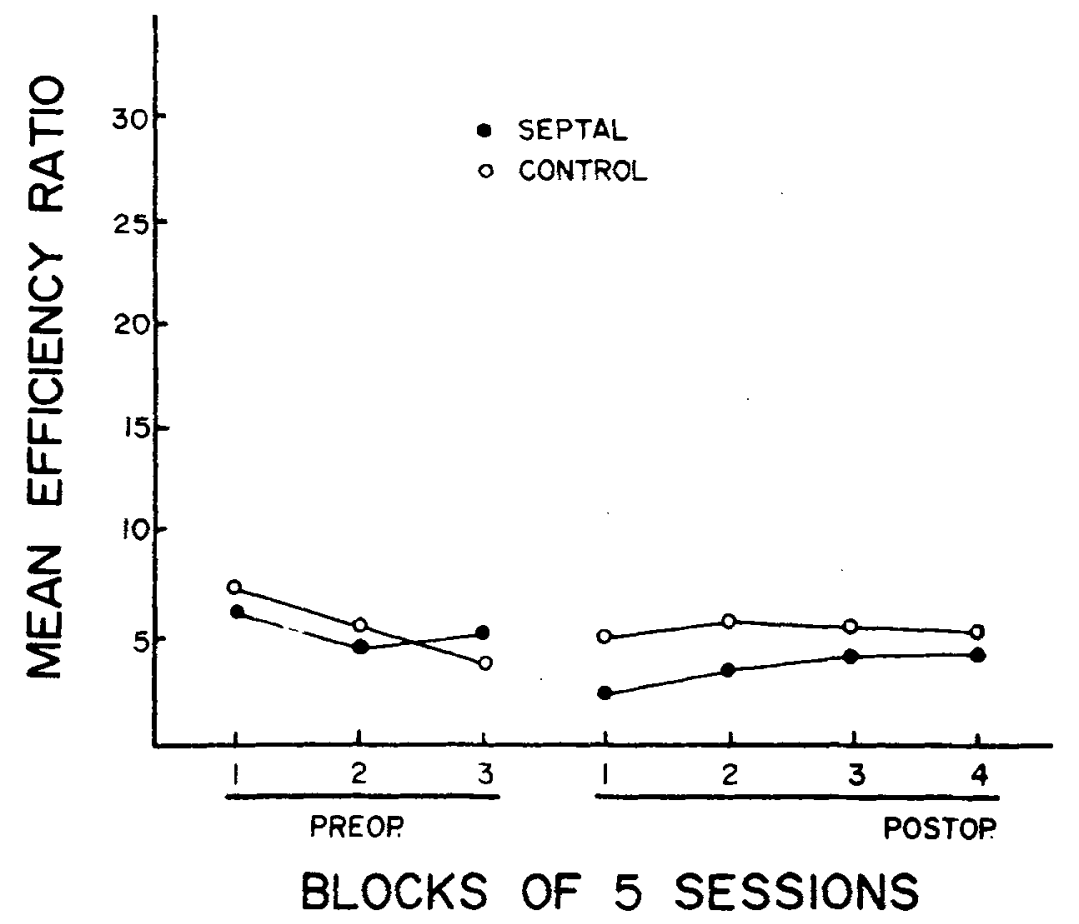

Fig. 1. Mean group efficiency (response/reinforcement) ratios under nondeprivation. The preoperative data points represent the first, middle, and last block of five sessions prior to surgery. 


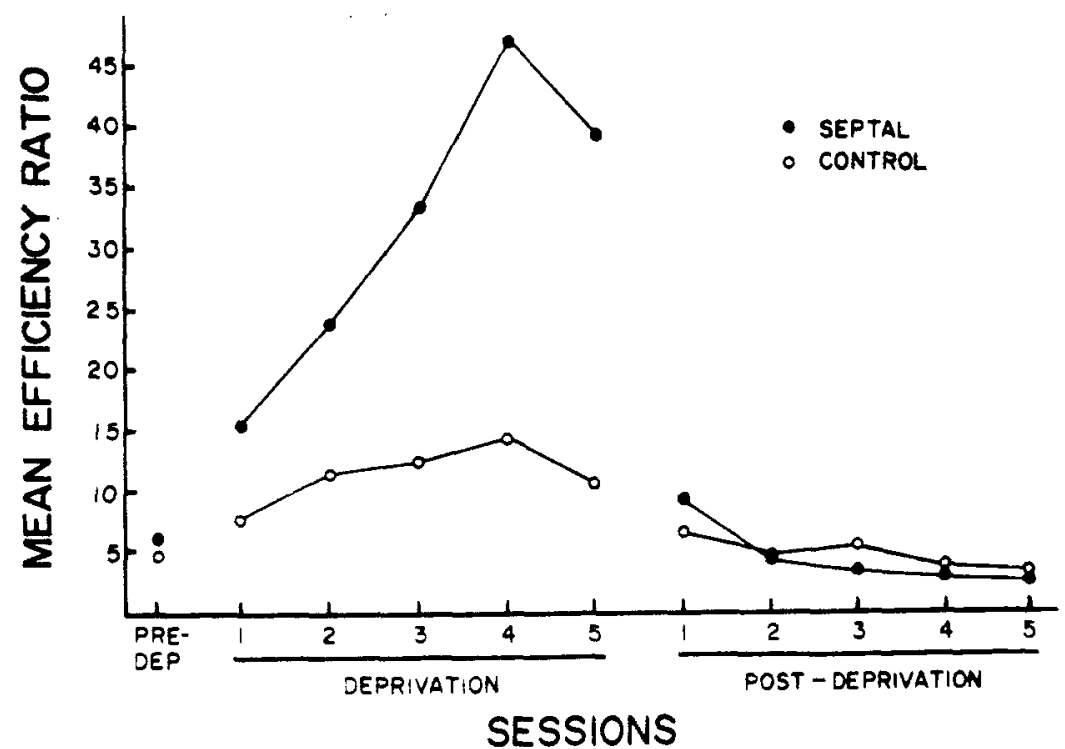

Fig. 2. Mean group efficiency ratios for the last session prior to deprivation, for the five deprivation sessions, and for the five sessions after deprivation.

electrode was lowered only $1.50 \mathrm{~mm}$, and no current was passed for the control S. Cortical aspirations were made under a dissecting microscope with a suction pipette.

Following testing, lesioned Ss were sacrificed with a lethal dose of sodium pentobarbital and were perfused with saline and $10 \%$ Formalin. Brains were then removed, fixed in Formalin, and embedded in celloidin. Sections in the area of the lesion were cut at 30 microns. Every fifth section was saved, mounted, and stained with cresyl violet for subsequent microscopic examination of lesion extent.

\section{RESULTS AND DISCUSSION}

Most of the septal lesions extended from just anterior to the genu of the corpus callosum (resulting in some cortical and callosal damage) to just anterior to the crossing of the anterior commissure. Bilateral destruction of the medial and lateral septal nuclei, nucleus accumbens septi, and the vertical limbs of the diagonal band was extensive in all Ss with septal lesions. Only one of the brains showed no damage to the septal nucleus triangularis and the dorsal fornix. Roughly equivalent amounts of tissue were ablated in the cortical control Ss, with slight unilateral damage occasionally occurring in the corpus callosum, cingulate gyrus, or dorsal hippocampus.

No postoperative differences were observed among the $\mathrm{Ss}$ in the control group on any of the measures taken. Data from Ss for which reliable fluid measures were obtained indicated no statistically significant effects of the each. While there was only minimal preoperative improvement on the DRL schedule, an analysis of variance indicated that the improvement was reliable $(p<.05)$. A Wilcoxon matched-pairs signed-ranks test on the last preoperative and first postoperative block of sessions indicated no changes in the performance of the control group, but a significantly lower ratio for the septal group $(p<.05)$. An overall analysis of variance of the postoperative data, however, failed to distinguish between the lesioned and control groups statistically.

The low ratios exhibited by the hamsters with septal lesions were due to an overall decrease in responding. Since saccharin intake and preference were not altered by the lesion, the response decrement was probably not due to reduced motivation for the reinforcement. Furthermore, a retention deficit seems improbable, since the lesioned Ss typically responded a few times at the beginning of the session. Observations of the animals showed that the septal Ss would then curl up and apparently go to sleep.

The results of placing the hamsters on water deprivation may be seen in Fig. 2. The septal group immediately showed large response increases, which are reflected in the high efficiency ratios. An analysis of variance on the efficiency ratios obtained during the deprivation sessions indicated a significant group effect $(p<.05)$, sessions effect $(p<.01)$, and a Lesion by Sessions interaction effect $(p<.05)$. When the animals were returned to the nondeprived condition (Fig. 2), the septal effect seen under deprivation immediately disappeared. represent the means of the first, middle, and last blocks of five sessions

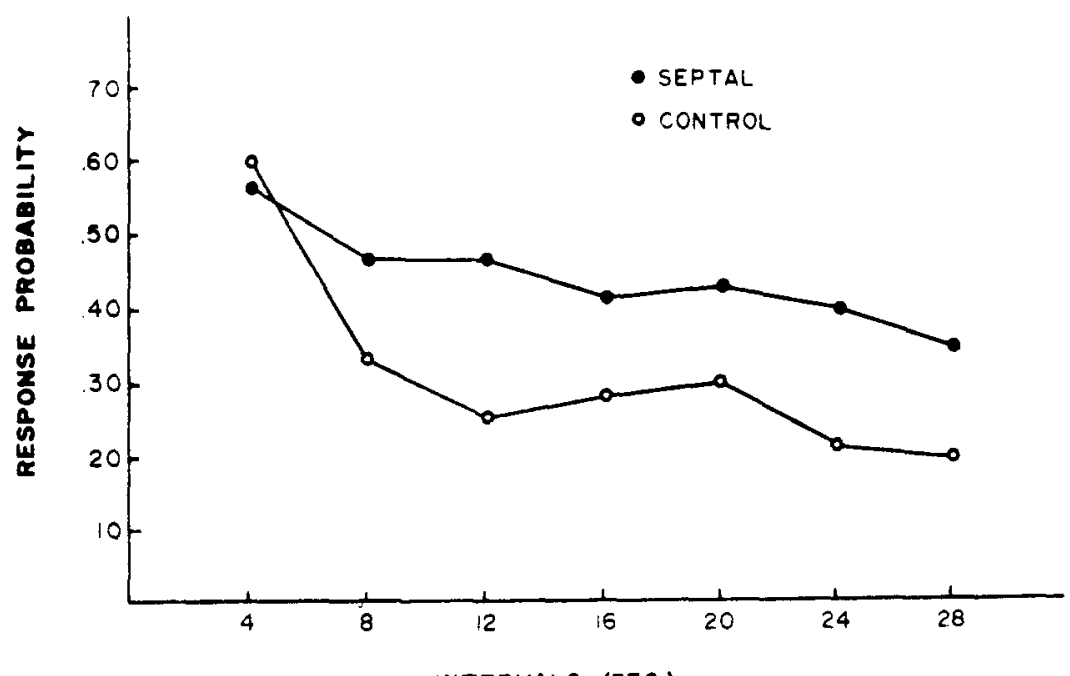

INTERVALS (SEC.)

Fig. 3. Mean group response probabilities (IRTs/OP) over seven 4-sec class intervals for the five water-deprivation sessions. 
An analysis of variance of these last five sessions showed no reliable group differences.

Interresponse time (IRT) data for the deprivation sessions were converted to IRTs per opportunity (IRTs/OP) (Anger, 1956), a measure computed by dividing the number of responses in each IRT category by the number of responses in that and all subsequent IRT categories. This transformation gives the conditional probability that a response will occur at a particular time given that the $S$ has paused at least that long since its previous response. Figure 3 shows the IRTs/OP for the two groups while under water deprivation. There is little evidence of temporal discrimination in either group, presumably due to the low response rates previously exhibited under nondeprivation. Somewhat surprisingly, the increased response probabilities of the lesioned group were found at all intervals except the shortest $(0-4 \mathrm{sec})$. Rats with septal lesions typically have much larger response probabilities than controls in the shorter interresponse intervals (e.g., Ellen, Wilson, \& Powell, 1964).

Like the septal rat, the septal hamster does have a pronounced deficit on the DRL schedule, even though the lesion does not result in increased fluid intake or saccharin preference. However, the deficit appears only under deprivation. This may be due to a masking of the DRL deficit by lesion-induced activity changes, since the lesioned hamsters were often observed sleeping during the sessions. Water deprivation may have served to increase activity (or arousal) and thus produce the increased responding on the DRL schedule. Gotsick (1969) has reported that rats given septal lesions were less active than controls under nondeprived conditions, except during the first $10 \mathrm{~min}$ of each hour-long session, while activity differences were largely obliterated under water or food deprivation.

Septal lesions in hamsters have been shown to increase activity levels during dark hours, but not in light-on hours (Hobbs, Miller, Bunnell, \& Peacock, 1972). To the extent that the results of the present study are due to lesion-produced activity changes, it should be possible to observe the DRL deficit in operated hamsters which are nondeprived, but run during the dark portion of their activity cycle.

ANGER, D. The dependence of interresponse times upon the relative reinforcement of different interresponse times. Journal of Experimental Psychology, 1956, 52, 145-161.

BURKETT, E. E., \& BUNNELL, B. N. Septal lesions and the retention of DRL performance in the rat. Journal of Comparative \& Physiological Psychology, $1966,62,468-471$.

CAREY, R. J. Contrasting effects of increased thirst and septal ablations on DRL responding in rats. Physiology \& Behavior, 1967a, 2, 287-290.

CAREY, R. J. Independence of effects of septal ablations on water intake and response inhibition. Psychonomic Science, $1967 \mathrm{~b}, 8,3-4$.

ELLEN, P., WILSON, A. S., \& POWELL, E. W. Septal inhibition and timing behavior in the rat. Experimental Neurology, $1964,10,120-132$.

GOTSICK, J. E. Factors affecting spontaneous activity in rats with limbic system lesions. Physiology \& Behavior, $1969,4,587-593$.

HARVEY, J. A., \& HUNT, N. F. Effect of septal lesions on thirst in rats as indicated by water consumption and operant responding for water reward. Joumal of Comparative \& Physiological Psychology. $1965,59,49-56$.

HOBBS, S. H., MILLER, C. R.. BUNNELL B. N., \& PEACOCK, L. J. General activity in hamsters with septal lesions. Physiology \& Behavior, 1972, in press.

MATALKA, E. S. The hoarding behavior and food intake of the hamster following hypothalamic and limbic forebrain lesions. $P h D$ dissertation. University of Florida, 1967.

SMITH, O. A., \& BODEMER, C. N. A stereotaxic atlas of the brain of the golden hamster (Mesocricetus auratus). Journal of Comparative Neurology, 1963, 120, 53-63. 